\title{
Mitochondrial localization of Dictyostelium discoideum dUTPase mediated by its N-terminus
}

\author{
Catherine P. Chia ${ }^{1 *}$ (D), Noriko Inoguchi ${ }^{2,3}$, Kyle C. Varon ${ }^{1}$, Bradley M. Bartholomai ${ }^{4}$ and Hideaki Moriyama ${ }^{1}$
}

\begin{abstract}
Objective: The nuclear and mitochondrial genomes of Dictyostelium discoideum, a unicellular eukaryote, have relatively high $\mathrm{A}+\mathrm{T}$-contents of $77.5 \%$ and $72.65 \%$, respectively. To begin to investigate how the pyrimidine biosynthetic pathway fulfills the demand for dTTP, we determined the catalytic properties and structure of the key enzyme deoxyuridine triphosphate nucleotidohydrolase (dUTPase) that hydrolyzes dUTP to dUMP, the precursor of dTTP.

Results: The annotated genome of $D$. discoideum identifies a gene encoding a polypeptide containing the five conserved motifs of homotrimeric dUTPases. Recombinant proteins, comprised of either full-length or core polypeptides with all conserved motifs but lacking residues 1-37 of the N-terminus, were active dUTPases. Crystallographic analyses of the core enzyme indicated that the C-termini, normally flexible, were constrained by interactions with the shortened $\mathrm{N}$-termini that arose from the loss of residues 1-37. This allowed greater access of dUTP to active sites, resulting in enhanced catalytic parameters. A tagged protein comprised of the N-terminal forty amino acids of dUTPase fused to green fluorescent protein (GFP) was expressed in D. discoideum cells. Supporting a prediction of mitochondrial targeting information within the N-terminus, localization and subcellular fractionation studies showed GFP to be in mitochondria. N-terminal sequencing of immunoprecipitated GFP revealed the loss of the dUTPase sequence upon import into the organelle.
\end{abstract}

Keywords: dUTPase, Dictyostelium discoideum, GFP, Mitochondrial targeting sequence

\section{Introduction}

The nuclear and mitochondrial genomes of Dictyostelium discoideum are $78 \%$ and $73 \% \mathrm{AT}$, respectively $[1,2]$, creating a substantial requirement for dUMP, the precursor for dTTP, during mitotic cell growth as well as during development when DNA replication also occurs [3-5]. To understand how the pyrimidine biosynthesis pathway accommodates the demand for dTTP, we began to focus on a key enzyme of the pathway, deoxyuridine triphosphate nucleotidohydrolase or dUTPase, which hydrolyzes dUTP to pyrophosphate and dUMP; dUMP is subsequently converted to dTTP. Concomitantly, a high

\footnotetext{
*Correspondence: cchia1@unl.edu

${ }^{1}$ School of Biological Sciences, Univ. Nebraska-Lincoln, Lincoln, NE 68588-0118, USA

Full list of author information is available at the end of the article
}

dTTP to dUTP ratio is ensured, thus minimizing the incorporation of uracil during DNA synthesis [6].

The curated genome of the soil amoeba $D$. discoideum shows a single gene (DictyBase Gene ID DDB_G0293374; [7]) predicted to encode a dUTPase polypeptide containing the five hallmark motifs (M1-M5) of homotrimeric dUTPases [8], seen in the alignments of the amino acid sequences from mustard, yeast and human (Fig. 1a). While the dUTPases of Arabidopsis thaliana and D. discoideum have substantial stretches of identity (73\%) within the 138-residue segment containing M1-M5 [9], their $\mathrm{N}$-termini have very low sequence similarity to each other, and to the human and yeast N-termini. Notably, within the lengthy $\mathrm{N}$-terminus of the $D$. discoideum dUTPase, atypical of most dUTPases, computational analyses predict a mitochondrial targeting sequence (MTS).

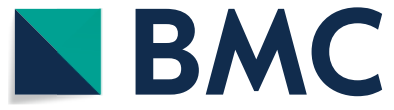

(c) The Author(s) 2020. This article is licensed under a Creative Commons Attribution 4.0 International License, which permits use, sharing, adaptation, distribution and reproduction in any medium or format, as long as you give appropriate credit to the original author(s) and the source, provide a link to the Creative Commons licence, and indicate if changes were made. The images or other third party material in this article are included in the article's Creative Commons licence, unless indicated otherwise in a credit line to the material. If material is not included in the article's Creative Commons licence and your intended use is not permitted by statutory regulation or exceeds the permitted use, you will need to obtain permission directly from the copyright holder. To view a copy of this licence, visit http://creativeco mmons.org/licenses/by/4.0/. The Creative Commons Public Domain Dedication waiver (http://creativecommons.org/publicdomain/ zero/1.0/) applies to the data made available in this article, unless otherwise stated in a credit line to the data. 


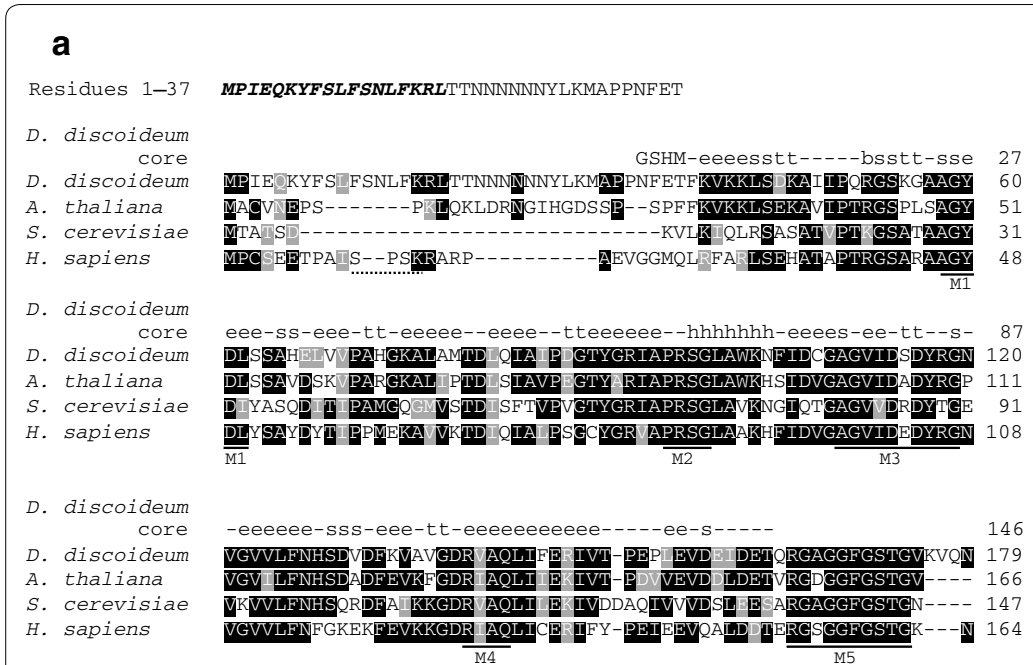

d

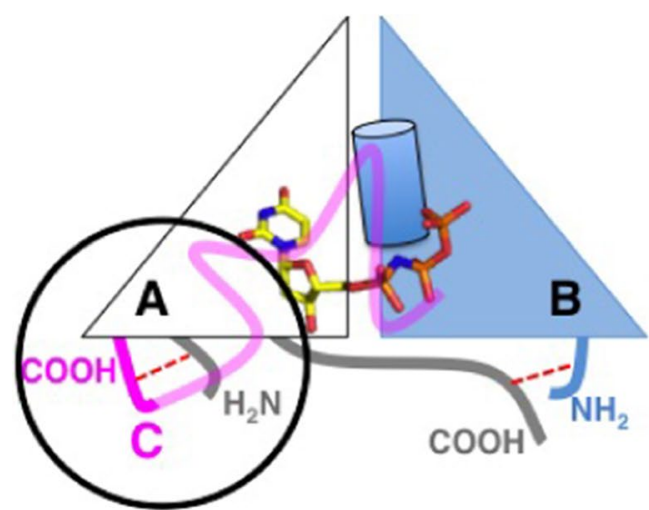

b

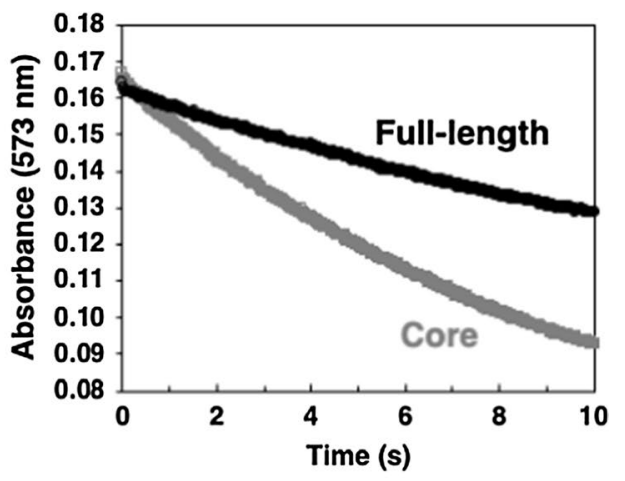

C

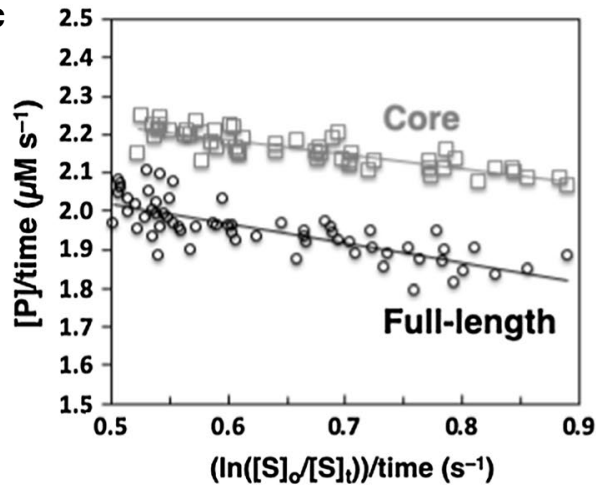

Fig. 1 Recombinant full-length and core proteins were active dUTPases. a Alignment of polypeptide subunit sequences of homotrimeric dUTPases from eukaryotes and location of conserved motifs. Sequences used are: Dictyostelium discoideum (UniProt ID, Q54BW5), Arabidopsis thaliana (Q9STG6), Saccharomyces cerevisiae (P33317), Homo sapiens nuclear isoform 2, nuclear type (P33316-2). The human mitochondrial dUTPase isoform is not shown due to the lack of sequence similarity between its the $\mathrm{N}$-terminal 69-residue targeting sequence and the D. discoideum $\mathrm{N}$-terminus. The N-terminal Gly-Ser-His-Met (GSHM) of the D. discoideum core dUTPase is a result of the cloning process. Dashes (-) in sequences are alignment gaps by MAFFT [27] and the graphical output was generated by BoxShade [28]. In the human dUTPase, the sequence SPSK (dotted underline) is a consensus sequence for phosphorylation [29]. M1-M5 are five conserved motifs (solid underlines) in homotrimeric dUTPases [8]. The secondary structure composition of chain B in the $D$. discoideum core dUTPase is shown by lowercase letters in the top line. These were identified by the DSSP in the 3D-structure (PDB ID 5F9K) $[30,31][29,30]:{ }^{\prime} h$ ' $=a$-helix; ' $b$ '= residue in isolated $\beta$-bridge; 'e' $=$ extended strand; ' $t$ ' $=$ turn; and 's' $=$ bend. Separately and above the alignment are shown residues 1-37 absent from the D. discoideum core dUTPase with a predicted MTS in bold italics [1517]. $\mathbf{b}$ Estimation of kinetic parameters of recombinant full-length and core dUTPases. Example data sets (one of five independent measurements each) from stopped-flow spectroscopy used to monitor the decreasing absorbance of cresol red from protons released during hydrolysis of dUTP by either full-length (black) or core (gray) dUTPase, each at $0.15 \mu \mathrm{M}$. c Transformed absorbance data of Panel b yielded values for $V_{\max }$ and $K_{M}$ of the full-length and core dUTPases (see Table 1) [32, 33]. d. Schematic illustration of the constrained orientations of the C-termini of Chains A and C of the core dUTPase. Triangles represent Chains A (white) and B (blue). A red dashed line shows the interaction between the C-terminus of Chain A (grey) and the $\mathrm{N}$-terminus of Chain B (blue). Also shown with a red dashed line is the interaction between the C-terminus of Chain C (solid pink) and the $\mathrm{N}$-terminus of Chain A (grey). This circled region is shown in more detail in Additional file 3: Fig. S3. Due to the lack of electron density, the C-terminus of Chain C (light pink) represents the region modeled on the high sequence identity to the $A$. thaliana dUTPase with its completed C-terminal coordinates (PDB ID 4OOP)

\section{Main text}

\section{Results}

A single gene codes for an active enzyme in the homotrimeric dUTPase family

To characterize the unusual $D$. discoideum dUTPase, we established first that the recombinant protein was an active dUTPase and determined its kinetic parameters. Also catalytically-active was a core version comprised of polypeptide subunits that retain M1-M5 but lack residues 1-37 of the N-terminus. A homotrimeric structure of the core dUTPase enzyme was confirmed by crystallographic analyses that additionally showed interactions of 
the shorter $\mathrm{N}$-termini with the $\mathrm{C}$-termini likely enhanced substrate access of the dUTP substrate to the active sites.

Recombinant His-tagged dUTPase proteins were expressed in E. coli, purified by metal-chelating chromatography and after removal of the His-tag, assayed for activity by measuring the release of protons with the $\mathrm{pH}$ indicator cresol red (Additional file 1: Text S1). As determined by the kinetic parameters calculated from stopped-flow measurements (Fig. 1b, c and Table 1), the $\mathrm{k}_{\text {cat }} / \mathrm{K}_{\mathrm{M}}$ of the core protein, lacking $\mathrm{N}$-terminal residues 1-37 (Fig. 1a), was 60-fold greater than that of the fulllength species, indicating the core was a more efficient enzyme.

Activity assays using an end-point method showed the full-length and core dUTPases both were metal-dependent enzymes inhibited by EDTA. $\mathrm{Mg}^{2+}$ was identified as the optimal divalent cation (Additional file 2: Table S1), as seen with homotrimeric dUTPases [6]. Both proteins specifically used dUTP ( $\leq 1 \%$ hydrolysis of other dNTPs; data not shown) and exhibited optimal activities at $60^{\circ} \mathrm{C}$ and $\mathrm{pH} 8$ (Additional file 3: Fig. S1). The catalytically efficient core dUTPase indicated that the $37 \mathrm{~N}$-terminal residues were not essential for activity.

The crystal structure of the core dUTPase showed it to be a homotrimer, indicating that the absent $37 \mathrm{~N}$-terminal residues were dispensable for oligomer formation (Additional file 3: Fig. S2; PDB ID 5F9K). In most homotrimeric dUTPases, the C-termini, containing M5 which aids in coordinating the ligand at the active site, are flexible. In the core structure, PISA interaction analyses revealed that the shorter $\mathrm{N}$-termini interacted with the C-termini of adjacent subunits, constraining their engagement with their respective active sites. The interactions are schematically shown for Chains $\mathrm{A}$ and $\mathrm{C}$, and Chains B and A (Fig. 1d). The limited movement of the C-termini contributed to the different inhibitor coordinates in each active site of the core dUTPase (Additional file 3: Fig. S2, insets). Notable is the position of Inhibitor B. Interactions (hydrogen bonds and salt bridges) by three glutamates of the $\mathrm{C}$-terminus of Chain $\mathrm{C}$ with residues of the N-terminus of Chain A (E120::H3+F5, E123::K5 and E126::K9) produced the most displaced orientation compared to Inhibitors $\mathrm{A}$ and $\mathrm{C}$ (detailed in Additional file 3: Fig. S3). The C-termini, which act

Table 1 Calculated kinetic parameters of D. discoideum dUTPases

\begin{tabular}{lllll}
\hline & $\mathbf{K}_{\mathbf{M}}, \boldsymbol{\mu M}$ & $\mathbf{V}_{\mathbf{m a x}^{\prime}} \boldsymbol{\mu} \mathbf{M ~ s}^{-\mathbf{1}}$ & $\mathbf{k}_{\mathbf{c a t}}^{\mathbf{a}} \mathbf{s}^{-\mathbf{1}}$ & $\begin{array}{l}\mathbf{k}_{\mathbf{c a t}} / \mathbf{K}_{\mathbf{M}} \\
\mathbf{M M}^{-1} \mathbf{s}^{\mathbf{1}}\end{array}$ \\
\hline Core & $0.5 \pm 0.1$ & $1.4 \pm 0.06$ & 9.3 & 18.6 \\
Full-length & $1.0 \pm 0.2$ & $0.5 \pm 0.03$ & 3.3 & 0.3
\end{tabular}

$\mathrm{a}_{\mathrm{cat}}=\mathrm{V}_{\max } /\left[\mathrm{E}_{\mathrm{T}}\right] ;\left[\mathrm{E}_{\mathrm{T}}\right]=0.15 \mu \mathrm{M}$ normally as lids for the substrate binding pockets [10$12]$, instead were restricted in their movement, which may have allowed increased access of dUTP to the three active sites compared to the full-length protein with its extended $\mathrm{N}$-termini. We postulate this to be why the core enzyme was more catalytically efficient in vitro compared to the full-length dUTPase.

\section{The N-terminus of D. discoideum dUTPase contains a mitochondrial targeting sequence}

The role of the N-terminus of the $D$. discoideum dUTPase was explored first by expressing in Ax2 cells a full-length dUTPase with GFP at the C-terminus of the polypeptide subunit (Fig. 2a). By microscopy, the fusion protein was observed to localize to Mitotracker ${ }^{\mathrm{TM}}$-labeled mitochondria (Fig. 2b). This finding supported the hypothesis that dUTPase is a mitochondrial protein. It also raised the possibility that an MTS resides within the N-terminus. Contrary to expectations, nuclei did not display any GFP fluorescence (Fig. 2c). A helical wheel projection showed that $\mathrm{N}$-terminal residues 1-20 of dUTPase formed an amphipathic helix [13] (Fig. 2e), with a net positive charge, a characteristic common to presequences of imported mitochondrial proteins [14]. Programs predicting targeting sequences indicated a high probability of dUTPase to be located in mitochondria, when fungi were used in the organism category, with cleavage after Leu18 upon import into the organelle (BaCelLo [15], MitoFates [16], MitoProt II [17]).

As a test for the presence of an MTS within the N-terminus of dUTPase, we added the forty $\mathrm{N}$-terminal residues of dUTPase to GFP, producing $\mathrm{N}_{1-40}$-dUTPase-GFP (Fig. 2a). Like the full-length dUTPase-GFP fusion protein, the expressed $\mathrm{N}_{1-40}$-dUTPase-GFP co-localized with Mitotracker ${ }^{\mathrm{TM}}$-labeled mitochondria (Fig. 2d), reinforcing the prediction of an MTS within the N-terminus of dUTPase. The majority of the mitochondria had both GFP and Mitotracker ${ }^{\text {TM }}$ signals, but not all Mitotracker ${ }^{\text {TM }}$ stained mitochondria had a GFP signal. No fluorescence was observed in nuclei (data not shown).

Protein blots, of whole cell lysates and mitochondria prepared from cells expressing $\mathrm{N}_{1-40}$-dUTPase-GFP, were probed with antibodies against porin, a mitochondria-specific protein [18] or GFP (Fig. 2f). As expected, the signal for anti-porin was enhanced in the mitochondria preparation (upper panel). Observed also in mitochondria was a strong anti-GFP signal (lower panel) that corroborated the microscopy images, indicating that $\mathrm{N}_{1-40}$-dUTPase-GFP localized to mitochondria. The two anti-GFP signals observed both in the lysate and mitochondria were interpreted to be the entire $\mathrm{N}_{1-40}$-dUTPase-GFP, predicted to be $32.5 \mathrm{kDa}$ and a processed version of it, migrating at $27.5 \mathrm{kDa}$. The 


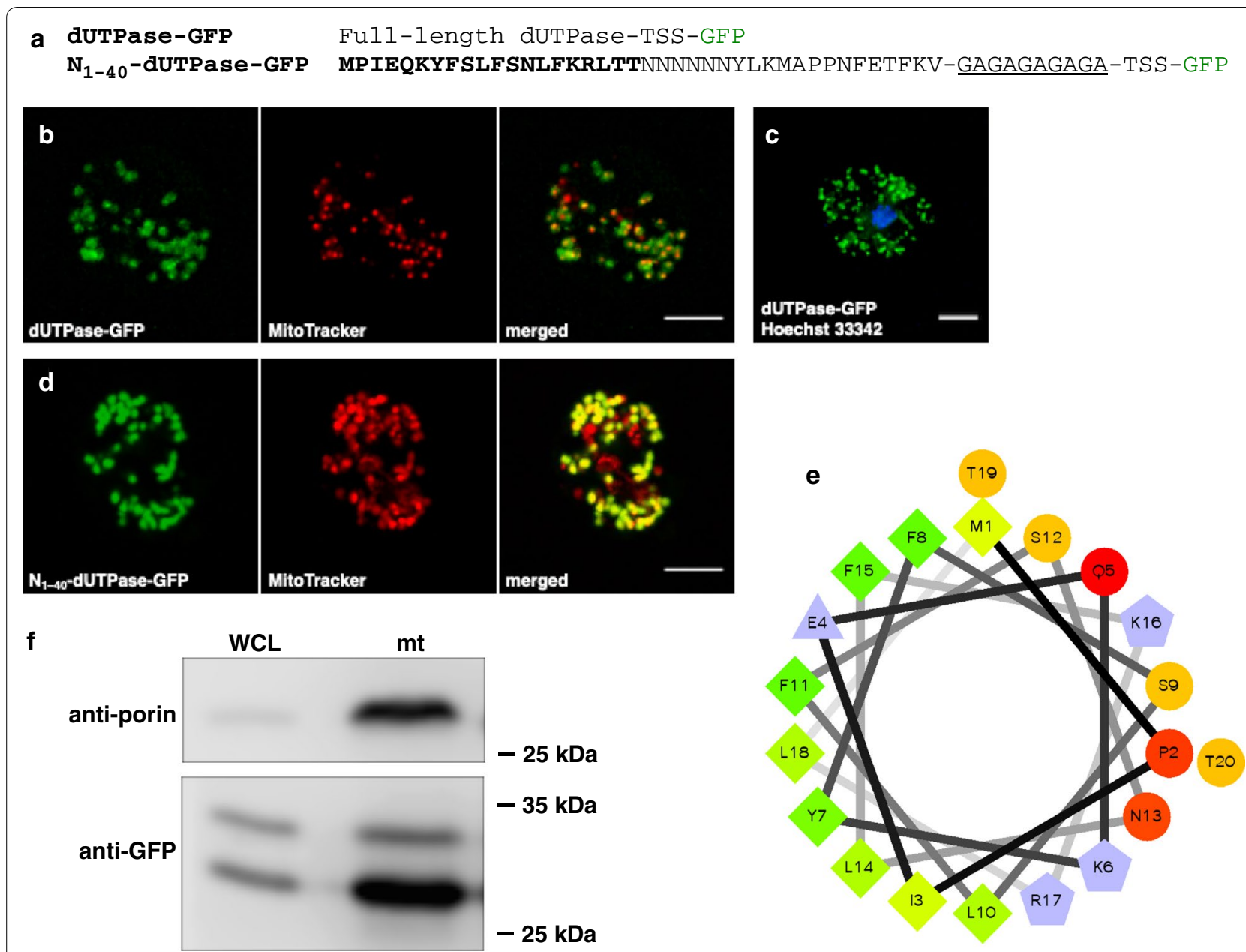

Fig. 2 dUTPase-GFP fusion proteins localized to mitochondria. a Sequences of expressed D. discoideum dUTPase-green fluorescent protein (GFP) fusions. The full-length $D$. discoideum dUTPase polypeptide had GFP fused to the C-terminal Asn (N) 179 to produce dUTPase-GFP. Residues 1-40 of dUTPase were fused to GFP with a (Gly-Ala) 5 linker; in bold are residues 1-20 used in the helical wheel drawn in Panel e. In both constructs, the Thr-Ser-Ser (TSS) tripeptide sequence arose from the cloning process. The underlined linker sequence was identified by N-terminal sequencing of protein immunoprecipitated by anti-GFP from Ax2 cells expressing $N_{1-40}$-dUTPase-GFP. $\mathbf{b}$ Confocal microscopy images of live Ax2 cells expressing dUTPase-GFP stained with Mitotracker; merged signals showed co-localization. Scale bar, 5 um. c Ax2 cells expressing dUTPase-GFP were fixed and stained with Hoechst 33342 to identify the nuclear DNA. dUTPase-GPF was absent from the nucleus. Scale bar, $5 \mu$ m. d Confocal microscopy images of live Ax2 cells expressing $N_{1-40}$-dUTPase-GFP stained with Mitotracker; merged signals showed co-localization of the GFP signal with mitochondria. Scale bar, 5 m. e Helical wheel of residues 1-20 of the $\mathrm{N}$-terminus of D. discoideum dUTPase shows its amphipathic character. Symbols represent the following: circles, hydrophilic residues; diamonds, hydrophobic residues; triangles, potentially negatively charged; and pentagons, potentially positively charged. Color code: the most hydrophobic residue is green, and the amount of green is decreasing proportionally to the hydrophobicity, with zero hydrophobicity coded as yellow; hydrophilic residues are coded red with pure red being the most hydrophilic (uncharged) residue, and the amount of red decreasing proportionally to the hydrophilicity; potentially charged residues are light blue [13]. $\mathbf{f}$ Immunoblots showed $\mathrm{N}_{1-40}$-dUTPase-GFP in mitochondria. A mouse monoclonal specific for D. discoideum porin (upper panel; $30.1 \mathrm{kDa}$ ) [18] or a rabbit polyclonal anti-GFP (lower panel) was used to probe blots of whole cell lysates (WCL; 100,000 cells) and enriched mitochondria preparations ( $\mathrm{mt} ; \sim 9 \times 10^{6}$ cell equivalents) from Ax2 cells expressing $\mathrm{N}_{1-40}$-dUTPase-GFP. Positions of prestained markers are shown to the right of each blot

approximate size difference of $5 \mathrm{kDa}$ corresponded to the predicted $4.8 \mathrm{kDa}$ mass of the forty residues of dUTPase fused to GFP, suggesting the loss of the dUTPase amino acids after import into the organelle.

To determine that cleavage of $\mathrm{N}_{1-40}$-dUTPase-GFP occurred, we obtained the $\mathrm{N}$-terminal sequence of the
$27.5 \mathrm{kDa}$ protein immunoprecipitated from cell lysates using anti-GFP antibodies. The processed fusion protein was cleaved after Gly41, the first glycine of the (GlyAla) $)_{5}$ linker between the dUTPase and GFP sequences (Fig. 2a), confirming the loss of all forty dUTPase residues. Though within the range of presequence lengths, 
this was lengthier than the predicted presequence that identified cleavage after Leu18, a residue common within an R-2 motif recognized by the mitochondrial processing peptidase $[16,19]$ (reviewed in 14). An intermediate was not detected by immunoblotting, but it is possible that the fusion protein was cleaved twice, once after Leu18 and then after Gly41, generating the final $A(G A)_{4}$-GFP molecule inside the mitochondria.

\section{Summary}

Activity and structural analyses of the $D$. discoideum dUTPase showed it to be a functional enzyme with attributes typical of most eukaryotic dUTPases. The unusually long $\mathrm{N}$-terminus of the polypeptide subunit was found to be dispensable for catalytic activity as well as homotrimer formation. Microscopy and biochemical evidence showed that fusion proteins, where GFP was attached to the full-length polypeptide or the forty $\mathrm{N}$-terminal amino acids of dUTPase, localized to mitochondria, but not to nuclei, indicating the presence of an MTS within the $\mathrm{N}$-terminus of the $D$. discoideum dUTPase.

\section{Limitations}

The presence of dUTPase in $D$. discoideum mitochondria is reasonable given the needed synthesis of nucleotides for replication of the organellar DNA that is $73 \%$ AT. Except for the human enzyme, however, no other dUTPase has been reported to be in mitochondria. Based on its nuclear presence in yeast, flies and humans [20-23], we also expected to see dUTPase in nuclei, but did not observe a GFP signal in nuclei of $D$. discoideum cells of log-phase cultures expressing the full-length dUTPaseGFP fusion protein (Fig. 2c). Technical reasons could account for an absence of a nuclear signal. Fused to the C-terminus and larger than an individual dUTPase subunit (mass of the predicted full-length polypeptide is $19.8 \mathrm{kDa})$, the GFP sequence $(27.6 \mathrm{kDa})$ may have interfered with homotrimer assembly or masked nuclear localization signals. The successful cytological detection of dUTPase in the nucleus may require a construct with a small tag that is less likely to disrupt folding or cover signal epitopes on the dUTPase surface, coupled with methods that synchronize cell division or identify cells entering S-phase when presumably the nucleotide biosynthesis enzymes are actively providing dNTPs for DNA replication.

Alternatively, a nuclear-localized dUTPase in D. discoideum may be a location-specific isoform, rather than the predicted full-length protein. The human dUTPase has nuclear and mitochondrial isoforms produced from the same gene through the use of alternative $5^{\prime}$ exons $[23$, 24]. In Drosophila, two transcripts for dUTPase also are produced from a single gene, resulting in two isoforms with different $\mathrm{N}$-termini [20]. Depending on the tissue and developmental stage, the isoforms are present either in the nucleus or the cytoplasm; neither is found in mitochondria. Currently, there are no data (such as cDNAs) pointing to $D$. discoideum dUTPase isoforms, but these cannot be ruled out as there are examples in $D$. discoideum where two versions of a protein are generated from single copy genes. These include the peroxisomal and mitochondrial isoforms of the enzyme acetoacetyl-CoA thiolase (DDB_G0271544) that arise from alternative start codons [25], and (cytoplasmic and mitochondrial) isoforms of fumarase (DDB_G0280495), inferred from expressed sequence tag data. Fumarase isoforms have been documented extensively in yeast and mammals (reviewed in 26).

An increasing number of eukaryotic proteins are reported to have more than one subcellular location, and enzymes of nucleotide synthesis pathways are candidates for multiple locations since DNA replication occurs in the nucleus, mitochondria and chloroplasts. The mitochondrial location of the $D$. discoideum dUTPase, and the previously identified mitochondrial and nuclear isoforms of the human dUTPase, raises questions of whether other enzymes in the pyrimidine nucleotide biosynthesis pathway are similarly positioned and whether other eukaryotes share this pattern of multiple locations. The genetically-tractable $D$. discoideum is an experimental system that can be exploited to determine and explore molecular mechanisms of the subcellular distribution of thymidylate synthase, dCMP and dCTP deaminases (supported by expressed sequence tag data), and should reveal whether these enzymes use targeting strategies that coordinate their distribution with that of dUTPase, ensuring dNTPs for the replication of nuclear and organellar DNA.

\section{Supplementary information}

Supplementary information accompanies this paper at https://doi. org/10.1186/s13104-019-4879-7.

Additional file 1: Text S1. Methods

Additional file 2. Metal-dependence and statistics for refinements.

Additional file 3: Figure S1. Temperature and pH optima. Figure S2. Secondary structure \& altered positions. Figure S3. Interactions between Chains $A$ and $C$.

Abbreviations

dUTPase: deoxyuridine triphosphate nucleotidohydrolase; GFP: green fluorescent protein; MTS: mitochondrial targeting sequence.

\section{Acknowledgements}

This research used resources from the Advanced Photon Source, a US Department of Energy (DOE) Office of Science User Facility operated for the DOE Office of Science by Argonne National Laboratory under Contract No. DE-AC02-06CH11357. We thank: Dr. Javier Seravalli (Dept. of Biochemistry, UNL) for his contributions to the stopped-flow activity assays; Dr. Jay Dunlap, 
Dr. Jennifer Loros, and the Microscopy Core (Dartmouth) for allowing BMB to carry out follow-up microscopy imaging for this project while pursuing his Ph.D. thesis work.

\section{Authors' contributions}

CPC wrote the manuscript with major contributions to the crystallography analyses from $\mathrm{NI}$ and $\mathrm{HM}$. NI and HM performed crystallography and analyses of the 3D structure of the core dUTPase. BB performed microscopy imaging and assisted CPC with DNA cloning. CPC and KCV purified proteins. CPC, $\mathrm{HM}$ and KV measured and calculated enzyme activities. All authors read and approved the final manuscript.

\section{Funding}

The John C. and Nettie V. David Memorial Trust Fund through a UNL Research Council Grant-in-Aid to CPC provided partial support of this work. B. Bartholomai received support from the UCARE Program at UNL and a Grant in Aid of Research from Sigma Xi.

\section{Availability of data and materials}

The structure of the $D$. discoideum core dUTPase was deposited in the Protein Data Bank with the ID 5F9K. The dataset supporting the conclusions of this article are included within the article and additional materials.

\section{Ethics approval and consent to participate}

Not applicable.

\section{Consent for publication}

Not applicable.

\section{Competing interests}

The authors declare that they have no competing interests.

\section{Author details \\ ${ }^{1}$ School of Biological Sciences, Univ. Nebraska-Lincoln, Lincoln, NE 68588-0118, USA. ${ }^{2}$ Department of Biological Sciences, Univ. Alabama in Huntsville, Huntsville, AL 35899, USA. ${ }^{3}$ iXpressGenes Inc, Hudson Alpha Dartmouth College, Hanover, NH 03755, USA. \\ Received: 8 August 2019 Accepted: 26 December 2019 \\ Published online: 07 January 2020} Institute for Biotechnology, 601 Genome Way, Huntsville, AL 35806, USA. ${ }^{4}$ Present Address: Geisel School of Medicine, Dept. Molecular and Systems Biology,

\section{References}

1. Eichinger L, Pachebat JA, Glockner G, Rajandream MA, Sucgang R, Berriman M, et al. The genome of the social amoeba Dictyostelium discoideum. Nature. 2005;435(7038):43-57.

2. Ogawa S, Yoshino R, Angata K, Iwamoto M, Pi M, Kuroe K, et al. The mitochondrial DNA of Dictyostelium discoideum: complete sequence, gene content and genome organization. Mol Gen Genet. 2000;263(3):514-9.

3. Chen G, Shaulsky G, Kuspa A. Tissue-specific G1-phase cell-cycle arrest prior to terminal differentiation in Dictyostelium. Development. 2004;131(11):2619-30.

4. Muramoto T, Chubb JR. Live imaging of the Dictyostelium cell cycle reveals widespread S phase during development, a G2 bias in spore differentiation and a premitotic checkpoint. Development. 2008;135(9):1647-57.

5. Shaulsky G, Loomis WF. Mitochondrial DNA replication but no nuclear DNA replication during development of Dictyostelium. Proc Natl Acad Sci USA. 1995;92(12):5660-3.

6. Vertessy BG, Toth J. Keeping uracil out of DNA: physiological role, structure and catalytic mechanism of dUTPases. Acc Chem Res. 2009:42(1):97-106.

7. Fey P, Dodson RJ, Basu S, Chisholm RL. One stop shop for everything Dictyostelium: dictyBase and the Dicty Stock Center in 2012. Methods Mol Biol. 2013;983:59-92.

8. McGeoch DJ. Protein sequence comparisons show that the 'pseudoproteases' encoded by poxviruses and certain retroviruses belong to the deoxyuridine triphosphatase family. Nucleic Acids Res. 1990;18(14):4105-10.

9. Altschul SF, Madden TL, Schaffer AA, Zhang J, Zhang Z, Miller W, et al. Gapped BLAST and PSI-BLAST: a new generation of protein database search programs. Nucleic Acids Res. 1997;25(17):3389-402.

10. Mol CD, Harris JM, McIntosh EM, Tainer JA. Human dUTP pyrophosphatase: uracil recognition by a beta hairpin and active sites formed by three separate subunits. Structure. 1996;4(9):1077-92.

11. Persson R, Cedergren-Zeppezauer ES, Wilson KS. Homotrimeric dUTPases; structural solutions for specific recognition and hydrolysis of dUTP. Curr Protein Pept Sci. 2001;2(4):287-300.

12. Vertessy BG. Flexible glycine rich motif of Escherichia coli deoxyuridine triphosphate nucleotidohydrolase is important for functional but not for structural integrity of the enzyme. Proteins. 1997;28(4):568-79.

13. Zidovetzki R, Rost B, Armstrong DL, Pecht I. Transmembrane domains in the functions of Fc receptors. Biophys Chem. 2003;100(1-3):555-75. https://www.donarmstrong.com/cgi-bin/wheel.pl. Accessed 21 Dec 2019.

14. Mossmann D, Meisinger C, Vogtle FN. Processing of mitochondrial presequences. Biochem Biophys Acta. 2012;1819(9-10):1098-106.

15. Pierleoni A, Martelli PL, Fariselli P, Casadio R. BaCelLo: a balanced subcellular localization predictor. Bioinformatics. 2006;22(14):e408-16.

16. Fukasawa Y, Tsuji J, Fu SC, Tomii K, Horton P, Imai K. MitoFates: improved prediction of mitochondrial targeting sequences and their cleavage sites. Mol Cell Proteomics. 2015;14(4):1113-26.

17. Claros MG, Vincens P. Computational method to predict mitochondrially imported proteins and their targeting sequences. European journal of biochemistry/FEBS. 1996;241(3):779-86.

18. Troll H, Malchow D, Muller-Taubenberger A, Humbel B, Lottspeich F, Ecke $M$, et al. Purification, functional characterization, and CDNA sequencing of mitochondrial porin from Dictyostelium discoideum. J Biol Chem. 1992;267(29):21072-9.

19. Indio V, Martelli PL, Savojardo C, Fariselli P, Casadio R. The prediction of organelle-targeting peptides in eukaryotic proteins with grammatical-restrained hidden conditional random fields. Bioinformatics. 2013;29(8):981-8.

20. Bekesi A, Zagyva I, Hunyadi-Gulyas E, Pongracz V, Kovari J, Nagy AO, et al. Developmental regulation of dUTPase in Drosophila melanogaster. J Biol Chem. 2004;279(21):22362-70.

21. Ghaemmaghami S, Huh WK, Bower K, Howson RW, Belle A, Dephoure $\mathrm{N}$, et al. Global analysis of protein expression in yeast. Nature. 2003:425(6959):737-41.

22. Huh WK, Falvo JV, Gerke LC, Carroll AS, Howson RW, Weissman JS, et al. Global analysis of protein localization in budding yeast. Nature. 2003;425(6959):686-91.

23. Ladner RD, Caradonna SJ. The human dUTPase gene encodes both nuclear and mitochondrial isoforms. Differential expression of the isoforms and characterization of a CDNA encoding the mitochondrial species. J Biol Chem. 1997;272(30):19072-80.

24. Ladner RD, McNulty DE, Carr SA, Roberts GD, Caradonna SJ. Characterization of distinct nuclear and mitochondrial forms of human deoxyuridine triphosphate nucleotidohydrolase. J Biol Chem. 1996;271(13):7745-51.

25. Isezaki N, Sekiba A, Itagaki S, Nagayama K, Ochiai H, Ohmachi T. Dictyostelium acetoacetyl-CoA thiolase is a dual-localizing enzyme that localizes to peroxisomes, mitochondria and the cytosol. Microbiology 2015;161(7):1471-84.

26. Yogev $O$, Naamati A, Pines O. Fumarase: a paradigm of dual targeting and dual localized functions. FEBS J. 2011;278(22):4230-42.

27. Katoh K, Standley DM. MAFFT multiple sequence alignment software version 7: improvements in performance and usability. Mol Biol Evol. 2013;30(4):772-80.

28. Hofmann K, Baron M. BoxShade. https://embnet.vital-it.ch/software/ BOX form.html. Accessed 21 Dec 2019.

29. Ladner RD, Carr SA, Huddleston MJ, McNulty DE, Caradonna SJ. Identification of a consensus cyclin-dependent kinase phosphorylation site unique to the nuclear form of human deoxyuridine triphosphate nucleotidohydrolase. J Biol Chem. 1996;271(13):7752-7.

30. Kabsch W, Sander C. Dictionary of protein secondary structure: pattern recognition of hydrogen-bonded and geometrical features. Biopolymers. 1983;22(12):2577-637. 
31. Touw WG, Baakman C, Black J, te Beek TA, Krieger E, Joosten RP, et al. A series of PDB-related databanks for everyday needs. Nucleic Acids Res. 2015;43(Database issue):D364-8.

32. Inoguchi N, Chaiseeda K, Yamanishi M, Kim MK, Jang Y, Bajaj M, et al. Structural insights into the mechanism defining substrate affinity in Arabidopsis thaliana dUTPase: the role of tryptophan 93 in ligand orientation. BMC Res Notes. 2015;8(1):784.
33. Larsson G, Nyman PO, Kvassman JO. Kinetic characterization of dUTPase from Escherichia coli. J Biol Chem. 1996;271(39):24010-6.

\section{Publisher's Note}

Springer Nature remains neutral with regard to jurisdictional claims in published maps and institutional affiliations.
Ready to submit your research? Choose BMC and benefit from:

- fast, convenient online submission

- thorough peer review by experienced researchers in your field

- rapid publication on acceptance

- support for research data, including large and complex data types

- gold Open Access which fosters wider collaboration and increased citations

- maximum visibility for your research: over 100M website views per year

At BMC, research is always in progress.

Learn more biomedcentral.com/submissions 